\section{Rapport annuel EMH 2000}

Le concept existant des Editions Médicales Suisses EMH a été analysé et retravaillé au cours d'un séminaire fin 1999. Les résultats ont été consignés dans la "Vision 2001», qui fut présentée en février $2000 \mathrm{au}$ Conseil d'administration des EMH et unanimement adoptée par celui-ci. Par la suite, ce document fut également porté à la connaissance du Comité central de la FMH qui l'approuva. L'énorme engagement fourni par l'ancienne et de la nouvelle rédaction pour la restructuration de nos publications constitue un point essentiel de ce document. Les rédactrices et rédacteurs ont permis la création de journaux performants quant à la politique professionnelle, la formation médicale et les publications scientifiques. Ils méritent de grands remerciements pour leur travail.

Bien que les points essentiels de la "Vision 2001" aient déjà été publiés, ils sont rappelés ci-dessous:

- Le Bulletin des médecins suisses renonce à la publication d'articles de formation continue et se concentre sur des thèmes de politique professionnelle.

- Le Swiss Medical Weekly (ancien Journal Suisse de Médecine) paraît exclusivement en anglais, tous les quinze jours. Le but est de faire du SMW à moyen terme un journal d'audience internationale et d'augmenter son "impact factor", afin de proposer aux auteurs une publication attrayante de niveau européen dans le domaine de la science.

- Depuis janvier 2001, un nouveau magazine de formation continue paraît sous le nom de Swiss Medical Forum. La partie principale du SMF est traduite dans les différentes langues nationales et est publiée en grande partie comme journal autonome en français et en allemand.

- Un programme de formation permanente sur 3 ans est prévu pour le SMF. Il est publié sur papier et dans une deuxième phase sera accompagné d'un système interactif d'autoévaluation sur Internet.

- Une version électronique de l'Annuaire médical suisse est accessible également sur Internet.

- Le Comité central de la FMH reconnaît l'intérêt accru du nouveau concept des EMH pour ses membres. La FMH renonce à une partie de ses droits de licence de l'année 2001 pour le Bulletin des médecins suisses, respectivement appelle ses membres à verser une cotisation exceptionnelle pour le financement d'une partie des coûts entraînés.

- Dans le domaine électronique, un site d'accès adapté à la profession médicale sera mis en place sur Internet.

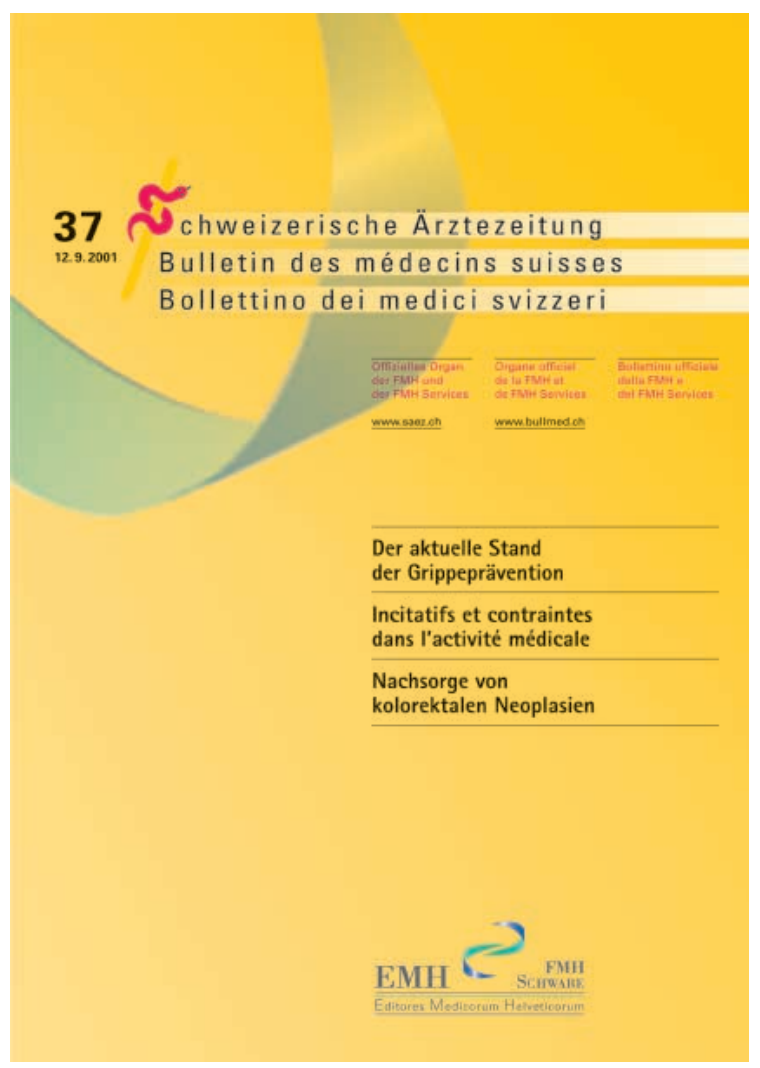

\section{Bulletin des médecins suisses}

Le Bulletin des médecins suisses (BMS) est toujours en mouvement! En effet, depuis qu'il est publié par les Editions médicales suisses son concept doit être revu et adapté en permanence aux nouvelles exigences. La période passée sous revue a été marquée par un besoin accru de la part du lectorat de disposer d'informations actuelles et fiables sur la politique professionnelle (quelque peu embrouillée par-fois), besoin au demeurant justifié. On peut dire que la nouvelle équipe décentralisée a démontré son efficacité à cet égard, avec pour rédacteur en chef le Dr Markus Trutmann, secondé par les rédacteurs externes: Dr Claude Aubert, Dr Ludwig T. Heuss, Reto Steiner et Dr Erhard Taverna qui remplissent cette tâche en sus de leurs activités professionnelles. La rédaction a aussi prouvé son indépendance rédactionnelle face à l'éditeur et aux EMH en pratiquant une politique rédactionnelle libérale, se voulant un reflet équilibré des opinions, le BMS étant une tribune où la diversité des opinions doit trouver place à côté de la voix officielle de la FMH.

Le BMS abandonne de publier des articles de formation continue. Cette "perte" peut toutefois être considérée comme une chance pour revaloriser la politique professionnelle et ses disciplines auxiliaires, telles que le droit, l'économie, l'éthique, l'histoire de la médecine, dans le BMS. La reconversion est prévue pour la fin 2001 et les travaux vont bon train.

Depuis le 21 juin 2000, le BMS est "en-ligne». Tous les articles paraissent dans leur intégralité simultanément à la version imprimée et sont accessibles gra- 
tuitement, à l'instar de ceux des autres revues EMH. Tous ceux aspirant à pouvoir consulter rapidement des documents, mais ne disposant pas forcément de place pour leur archivage, apprécieront cette innovation. En dépit de l'accroissement constant du nombre des utilisateurs, les possibilités d'interactions offertes par le forum électronique sur internet ne sont encore que trop timidement exploitées. Nonobstant cet état de choses ou plutôt à cause de cela, les éditeurs se proposent d'élargir l'offre dans le domaine de la publication électronique afin que chaque membre de la FMH puisse trouver ce qu'il souhaite.

En dépit du succès économique du BMS, il convient de remarquer qu'une partie substantielle de ses moyens est remise à la FMH et que les membres de la rédaction sont disponibles pour de nombreux autres projets. Une tâche pour les prochaines années est de développer les moyens de la rédaction, du point de vue matériel et en ce qui concerne le personnel.

\section{Swiss Medical Weekly}

Depuis plusieurs années, la nécessité de passer d'un journal trilingue présentant un mélange de travaux originaux et de formation permanente à un journal scientifique de niveau international en anglais se faisait sentir avec de plus en plus d'acuité. Ce changement, résolument préparé avec un grand labeur l'année passé, a été accompli en fin d'année. Toute la rédaction, avec son rédacteur en chef Prof. Andreas Schaffner, Zurich, et ses membres: Prof. Peter Gehr, Berne, Prof. André P. Perruchoud, Bâle, Prof. Werner

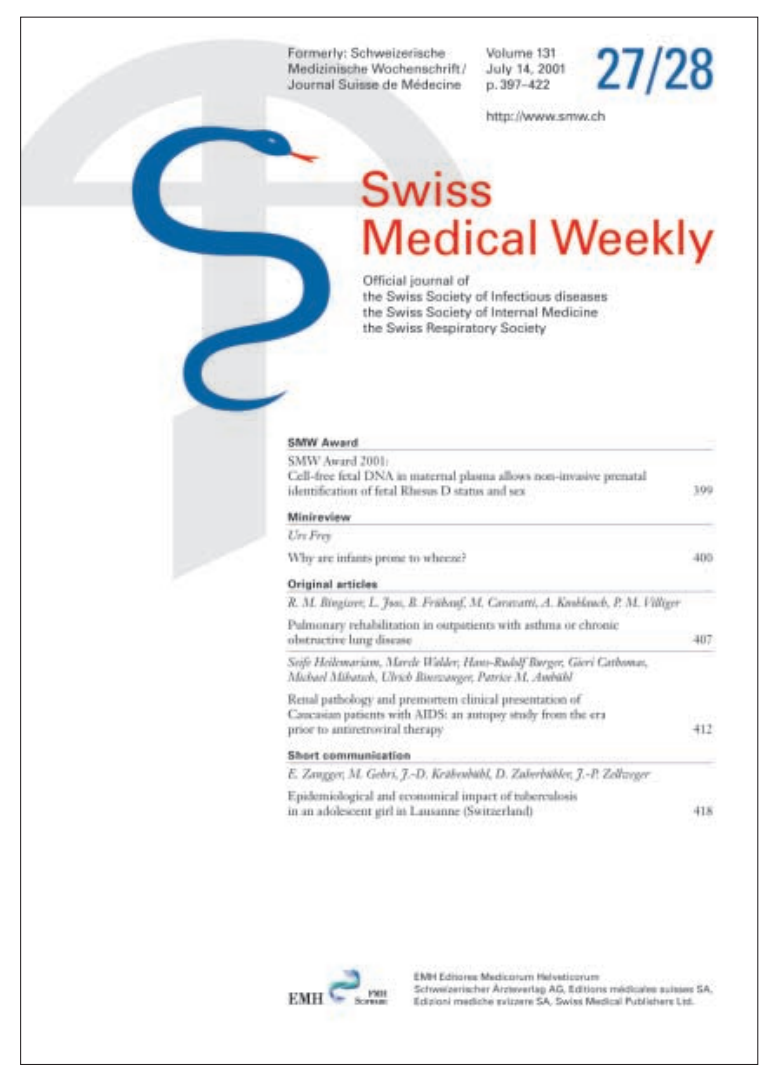

Straub, Berne, Prof. Peter Suter, Genève, et Prof. Ludwig von Segesser, Lausanne, a fourni un énorme travail. Elle est soutenue par un statisticien, des médecins et des traducteurs de langue maternelle anglaise au lectorat. La Drsse Natalie Marty coordonne la rédaction du SMW aux EMH. Gisela Wagner gère au secrétariat un système de peer-review efficace.

Chaque article est soumis au minimum à deux experts, dont l'un au moins à l'étranger.

Les articles sont aussi relus par le statisticien. Grâce aux moyens électroniques modernes, tous les membres de la rédaction sont en permanence en étroit contact.

Pour la première fois, la récompense SMW Award est mise à concours au niveau européen. Le SMW est disponible sur Internet depuis 1996 déjà.

Le SMW sera évidemment cité également dans la Medline et les autres banques de données médicales importantes, sous la nouvelle abréviation Swiss Med Wkly.

Le SMW perdra certainement en valeur économique, mais son but est de gagner en poids en tant qu'organe de publication scientifique.

\section{Swiss Medical Forum}

Il fallait un certain courage pour lancer un nouveau journal médical bilingue de formation continue sur un marché déjà bien saturé. L'argument décisif fut le concept convaincant prévoyant une collaboration étroite entre les médecins en cabinet et les médecins hospitaliers. Ce concept a mené à de nombreuses dis-

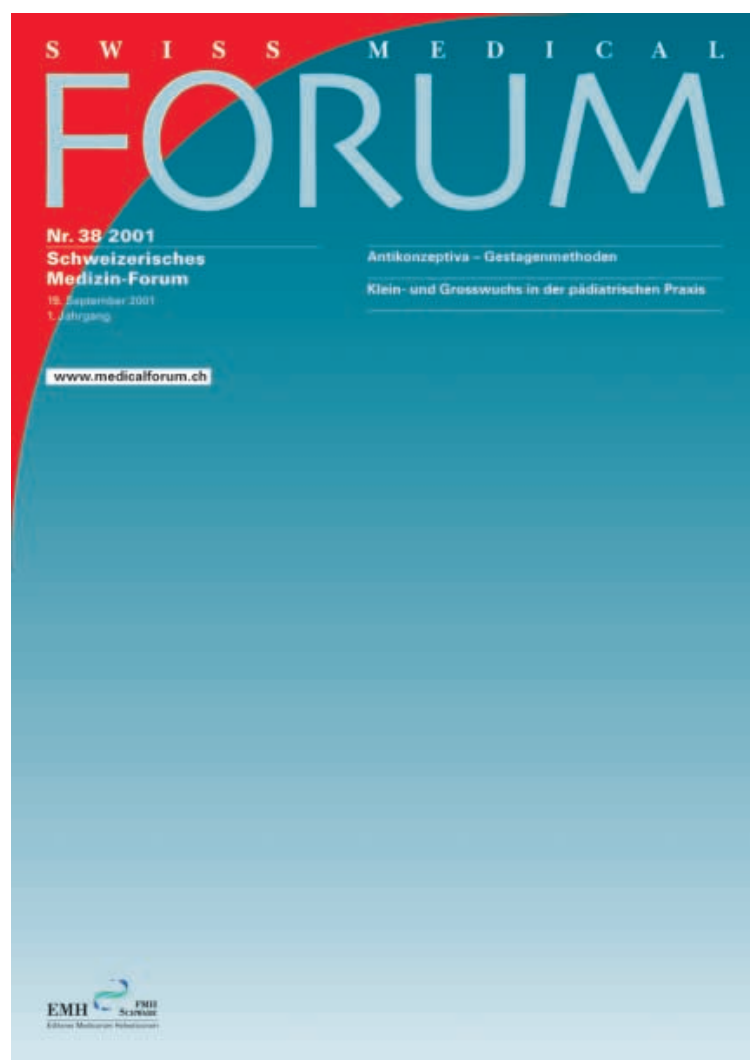


cussions très intéressantes et à une fructueuse collaboration dont il y a tout lieu de se réjouir. C'est le lancement du Swiss Medical Forum qui a permis de prendre en main les changements du SMW avec les conséquences économiques qui en résultaient. C'est pourquoi les droits d'éditeur de cette publication sont auprès des Editions Schwabe qui en mettent la licence à disposition des EMH, comme pour le SMW.

L'équipe de rédaction, avec son rédacteur en chef le Prof. Reto Krapf, et ses collaborateurs, les Dr Ludwig T. Heuss, Dr Pierre Périat, Prof. Ruedi Ritz, Prof. Rolf A. Streuli, Prof. Antoine de Torrenté et Prof. Bruno Truniger, a mis au point un concept probant et passionnant et a déjà pris contact avec des centaines d'auteurs. Dans sa phase de mise en place, le SMF était dirigé par le Dr Markus Trutmann avec Ruth Schindler, par la suite la Drsse Romaine Viollier s'est jointe à l'équipe et la complète actuellement en tant que Managing Editor et gère les affaires quotidiennes aux EMH.

La publication de la partie centrale du journal en deux éditions autonomes et complètes pour la Suisse francophone et germanophone s'est révélée une tâche difficile et demandant un grand investissement de travail. Le Dr Markus Trutmann a réussi à constituer une équipe de traduction compétente et fiable, capable de traduire des textes médicaux complexes dans l'une ou l'autre des langues. Le public réserve un accueil très favorable à ce nouveau journal. Tous les auteurs sollicités consentent volontiers à collaborer. L'étape suivante est l'autoévaluation sur Internet. Des travaux préparatoires intenses se déroulent actuellement sous la direction du Dr Markus Trutmann.

\section{PrimaryCare}

A l'origine du journal PrimaryCare se trouve une initiative de la Société Suisse de Médecine Générale (SSMG) qui souhaitait avec une publication propre, sous son influence, refléter les soucis et besoins des médecins de premiers recours. Le concept mis au point par les EMH trouva rapidement l'assentiment de la SSMG. Dans les négociations ultérieures, le contact positif avec la SSMG et le Collège de Médecine de Premier Recours (CMPR) porta ses fruits et c'est ainsi que fut fondé PrimaryCare, le journal de médecine de premier recours. PrimaryCare est l'organe officiel de publication de la SSMG et du CMPR.

Le journal est soutenu par une organisation simple et efficace qui permet aux différents partenaires (les EMH et les sociétés de premier recours) de faire valoir leur opinion. Le financement ouvert du journal est également exemplaire, il permet aux sociétés concernées de recevoir une participation aux bénéfices (voir PrimaryCare 2001;1:230-7).

PrimaryCare est dirigé par la Drsse Natalie Marty (rédactrice en chef), la Drsse Romaine Viollier (rédactrice en chef adjointe) et Louise Bigler (secrétariat). La rédaction très active est composée de 6 représentants et représentantes de la SSMG et de deux représentants du CMPR: Dr Heinz Bhend, Dr Silva

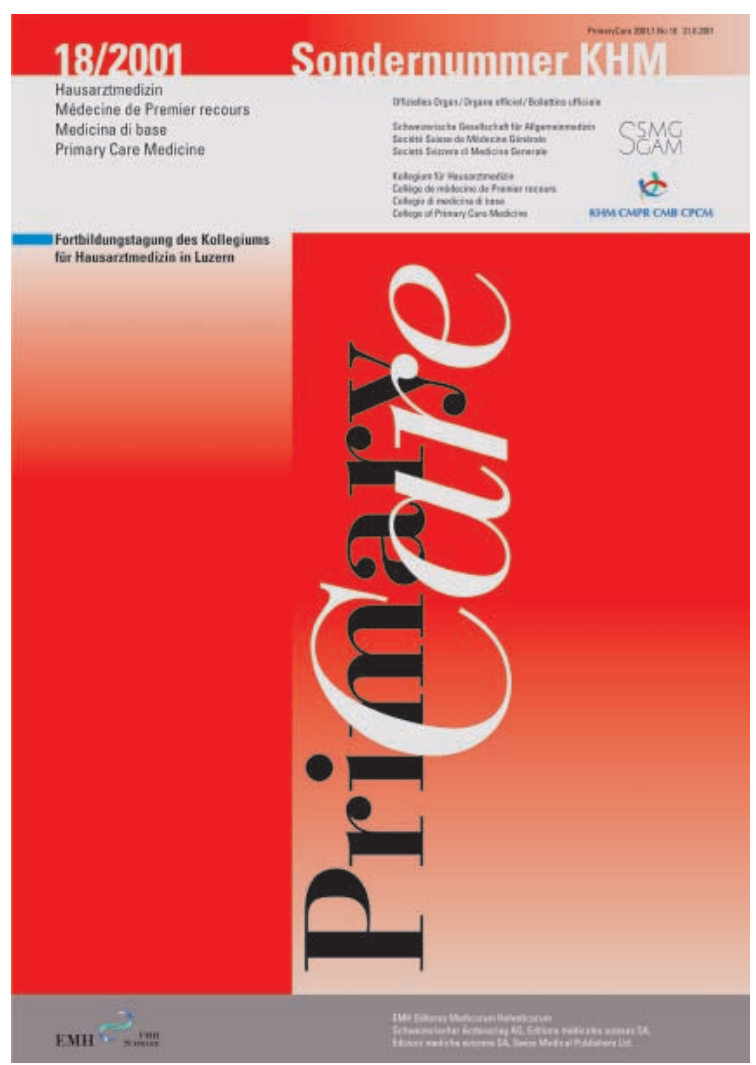

Keberle, Dr Bruno Kissling, Dr Pierre Klauser, Dr François Mottu, Dr Bernhard Rindlisbacher et Dr Daniel Widmer.

PrimaryCare paraît tous les 15 jours et publie des articles de politique professionnelle ainsi que des contributions touchant aux secteurs de la formation permanente et aux exigences de qualité. Le journal est devenu rapidement une plate-forme de discussion animée sur les questions et soucis des médecins de premier recours en Suisse. Par souci de crédibilité, la rédaction refuse par principe de publier des articles conçus en fonction d'annonces publicitaires. De même les articles de formation médicale ont comme objectif prioritaire l'indépendance et la neutralité.

\section{Annuaire médical suisse}

Pour la première fois l'Annuaire médical suisse n'existe plus en version imprimée uniquement mais aussi sur Internet. Il contient toutes les adresses des membres de la FMH, de la SSO et de la Société suisse des pharmaciens. La partie "hôpital» est entièrement révisée et complétée de nouvelles informations classées par service médical sur Internet. Un aspect très positif: la collaboration active des hôpitaux et des organisations de santé pour la livraison d'informations. Le projet en ligne a été lancé avec succès. Nous n'en sommes qu'à la phase de début du développement de ce produit électronique. Des informations peu connues jusqu'à présent deviennent accessibles et permettent d'intéressants sondages. Cependant, 


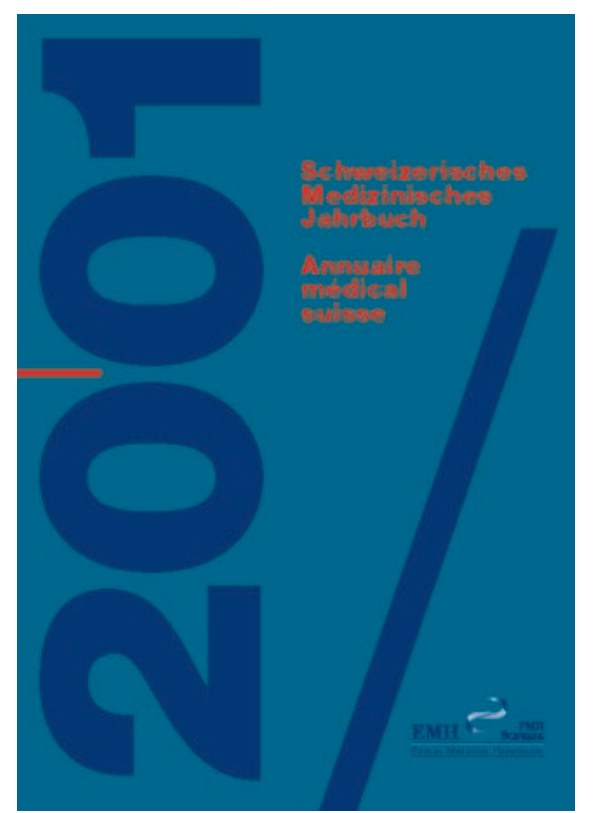

une grande subtilité est exigée pour l'usage de ces données, et les directives sur la protection des fichiers informatiques doivent être entièrement respectées.

\section{Médecine Cardiovasculaire}

Médecine Cardiovasculaire paraît depuis 1997 sous la direction de M. le Prof. Thomas F. Lüscher, Zurich, et de M. le Prof. René Lerch, Genève. Ce journal traite de tous les domaines de la médecine cardiovasculaire

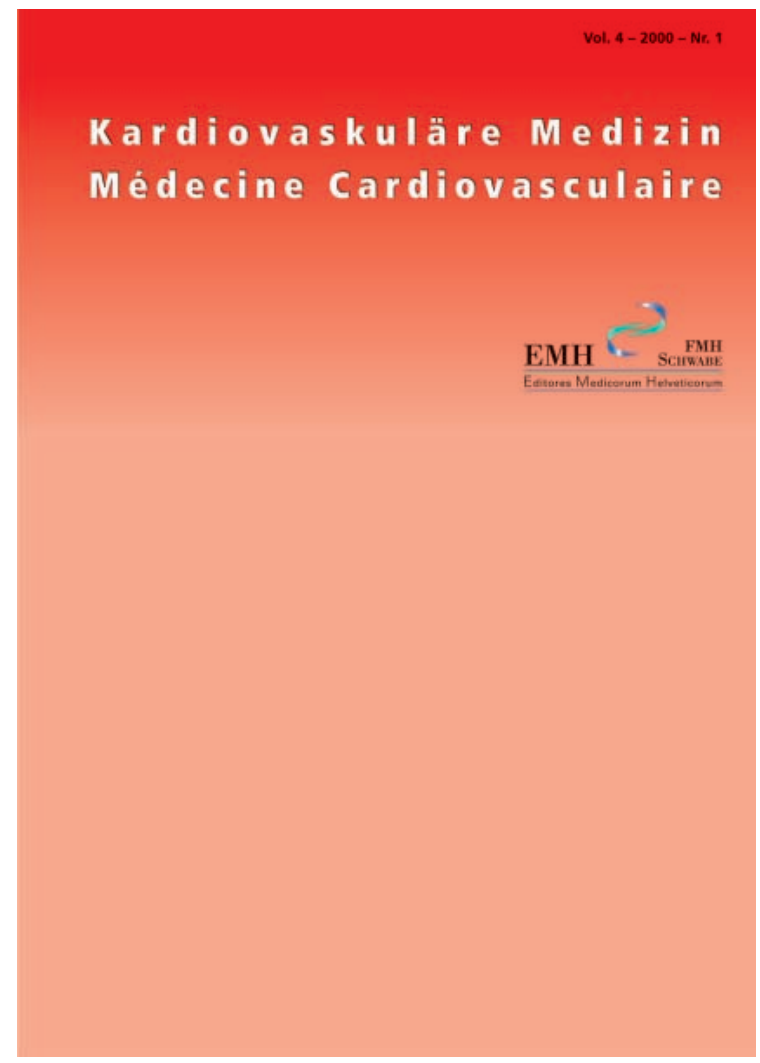

et constitue de ce fait un forum optimal pour la formation permanente, les travaux scientifiques dans le domaine de la cardiologie, de l'angiologie, de l'hypertonie, de la chirurgie cardiovasculaire. Outre des éditoriaux percutants, des travaux cliniques originaux et des comptes-rendus de congrès, le journal livre des informations intéressantes et, avec "images in cardiovascular medicine», met dans une certaine mesure les expériences de la pratique en pratique, avec des découvertes inhabituelles et en utilisant différents procédés visuels. Il s'y ajoute la rubrique "L'électrocardiogramme intéressant» et un calendrier des différentes sessions de formation professionnelle et congrès cardiologiques.

\section{Synapse}

Synapse est l'organe officiel de communication de la Société de médecine du Canton de Bâle-Campagne. Depuis cette année, le Dr Franz Rohrer remplace le Dr Lukas Wagner à la rédaction. Synapse est un support d'information politique, régionale et proche du public. Le soutien professionnel des EMH est acquis dans tous les domaines.

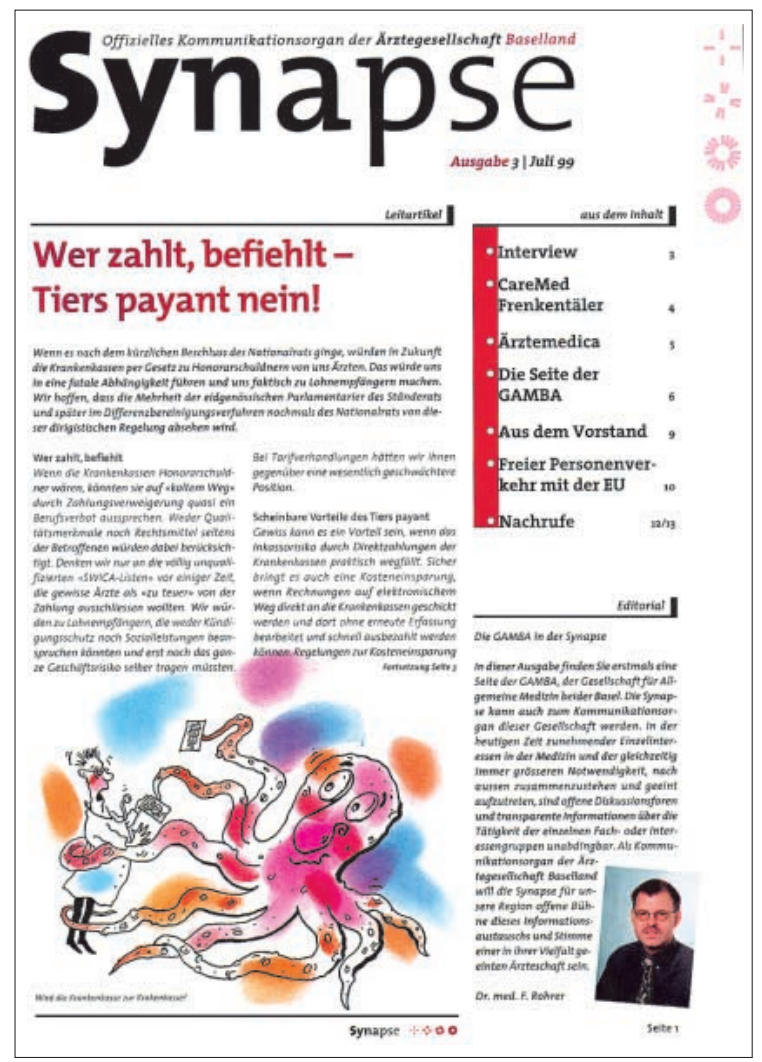

\section{medkalender}

Suite à la situation peu claire du prix des médicaments sur le marché, le medkalender ne put être publié comme prévu. Une nouvelle édition est planifiée. 


\section{Situation du personnel}

Avec un certain décalage dans le temps, tous les postes sont maintenant occupés. Le retard d'occupation de certains postes a conduit d'une part à des économies financières dans l'année rapportée et d'autre part à une surcharge de travail importante pour les collaborateurs et collaboratrices chargées de la mise en place. L'équipe des EMH est maintenant renforcée par une nouvelle lectrice (Dr Romaine Viollier) et une spécialiste du marketing et d'Internet (Natacha Gerber). De plus, les EMH forment un apprenti de commerce avec spécialisation en informatique, Roland Bienz.

\section{Projets de livre actuels}

Une nouvelle collection sur le thème de l'économie de santé est dirigée par le Dr Markus Trutmann. La collaboration de personnalités éminentes dans le domaine de l'économie de santé a pu être acquise. Le premier volume a paru (voir ci-contre).

Le «Manual der Schweizer Vertrauensärzte» est en réédition.

\section{EMH sur Internet}

Les activités du secteur Internet sont dominées par deux projets principaux: la mise en place du site EMH (www.emh.ch) qui a été réalisée dans les délais prévus fin 2000 après une phase de planification et de réalisation de 6 mois; et la présentation des nouvelles publications des Editions sur Internet.

Le site EMH sur Internet a été le projet le plus important de cette année, après la mise en ligne du Bulletin des médecins suisses (www.bullmed.ch) en juin 2000. Ce site réunit les différentes publications des EMH et couvre le besoin croissant en information générale. A long terme, le site EMH souhaite devenir le portail incontournable du sigle EMH pour les médecins suisses.

www.emh.ch propose dans la rubrique "News» en collaboration avec infomed des liens actuels avec les publications en texte intégral sur Internet. Les Editions infomed sont depuis des années une adresse sûre pour des informations fiables et nous nous réjouissons tout particulièrement d'avoir pu étendre notre fructueuse collaboration dans ce nouveau domaine. La caricature du jour, signée par un célèbre caricaturiste international, Randy Glasberger, est le clin d'œil quotidien que nous offrons aux visiteurs du site. La rubrique «News» doit encore être largement complétée, sans pourtant devenir un mélange désordonné de communiqués de presse.

Sous la rubrique "Medical addresses" se trouve la liste complète des membres de la FMH. Le formulaire joint à l'index des médecins permet de tenir la liste de la FMH à jour. Sous la rubrique "Hospitals", on peut consulter la liste des hôpitaux en Suisse, dé-
Jürg H. Sommer

Muddling Through Elegantly: Rationierung im Gesundheitswesen

taillée jusqu'au niveau des services. Cette base de donnée sur les hôpitaux est unique en Suisse et a été entièrement développée par les EMH.

Sous la rubrique "Congresses", on peut consulter les dates des congrès depuis la base de données sur les congrès des $\mathrm{EMH}$, que les lecteurs et lectrices connaissent comme supplément au Bulletin des médecins suisses. Les différentes manifestations peuvent être cherchées grâce à différentes fonctions. Un formulaire permet une inscription directe et sans frais supplémentaires dans le calendrier des congrès.

Sous "EMH interactive» se trouve un forum de discussion sur les différents thèmes de politique de santé. Dans le forum propre à chaque journal se trouvent également des commentaires et un courrier des lecteurs sur les articles publiés.

L'un des objectifs primordiaux de notre concept est de donner aux différentes publications une présentation de pointe sur Internet.

On trouve sous www.emh.ch des informations générales sur les différents journaux. Un lien renvoie aux pages correspondantes.

- Le Bulletin des médecins suisses est en ligne depuis le 21 juin 2000 (www.bullmed.ch). Les articles complets paraissent en même temps sur Internet et dans la version imprimée. Des fonctions de recherche élaborées donnent un caractère d'archives au site Internet.

- Le SMW (www.smw.ch) a été remodulé suite à un changement de nom et de concept. L'ancien Jour- 
nal Suisse de Médecine ou Schweizerische Medizinische Wochenschrift (devenu le Swiss Medical Weekly) a été l'un des premiers journaux en langue allemande à figurer sur Internet, et ce depuis 1996. Tous les articles depuis 1999 sont disponibles en version intégrale. Le nombre d'utilisateurs (environ 1000 par jour) est particulièrement élevé. Pour le nouveau SMW en anglais l'Internet est d'une importance primordiale. Nous suivons attentivement le développement des grandes banques de données médicales et nous intégrerons le SMW en parallèle à la Medline dans les banques de données de textes intégraux.

- La page du Swiss Medical Forum (www.medicalforum.ch) a été lancée en même temps que la version imprimée et propose de nombreuses fonc- tions de recherche intéressantes comme pour les autres titres EMH. D'une importance plus grande encore ici, le projet d'autoévaluation que nous sommes sur le point de lancer avec un logiciel particulier.

- Tous les articles du journal PrimaryCare sont accessibles à partir du site d'accueil de www. primarycare.ch: la rédaction collabore étroitement avec la rédaction du site de la SSMG (www. ssmg.ch).

On peut accéder à tous ces sites depuis la page www.emh.ch. Une liste de liens mise au point par la rédaction, la recherche directe dans la Medline et une boutique en ligne complètent la palette des possibilités offertes par Internet. 